\title{
Sexueller Sadismus: Aktueller Wissensstand und die Codierung gemäß DSM-5-TR und ICD-11
}

\author{
Andreas Mokros ${ }^{1}$ iD $\cdot$ Joachim Nitschke² \\ Eingegangen: 15. Dezember 2020 / Angenommen: 15. Dezember 2020 / Online publiziert: 6. Januar 2021 \\ (c) Der/die Autor(en) 2021
}

\section{Zusammenfassung}

Sexualsadismus bezeichnet eine lustvolle Erregbarkeit durch die Kontrolle, Erniedrigung oder Schmerzzufügung zulasten einer anderen Person. In den psychiatrischen Klassifikationskatalogen wird heute auf Zwang und Nichteinvernehmlichkeit abgestellt, um die forensische Ausprägung des Sexualsadismus von der konsensuellen Spielart im Sinne sadomasochistischer Rollenspiele abzugrenzen (,,bondage“ und Disziplinierung, Dominanz und Submission, Sadismus und Masochismus; kurz: BDSM). Ferner ist zwischen diesen Formen des Sexualsadismus und dem sog. Charakter- oder Alltagssadismus als Persönlichkeitszug zu unterscheiden. Im vorliegenden Beitrag wird die aktuelle Nosologie der forensisch-psychiatrisch bedeutsamen zwangsweisen sexuell-sadistischen Störung (,coercive sexual sadism disorder“) gemäß ICD-11 erläutert. Die Sexual Sadism Scale wird als diagnostisches Hilfsmittel für die gutachterliche Beurteilung vorgestellt. Befunde zur Kriminalprognose werden dargestellt, wobei die Diagnose per se nicht nennenswert mit erneuter Sexual- oder Gewaltdelinquenz nach Entlassung assoziiert ist, aber einen starken Zusammenhang mit äußerst schwerwiegenden Einweisungsdelikten zeigt (konkret: sexuell motivierte Tötungsdelikte). Wie aktuelle Literaturrecherchen ergeben, liegen aus den letzten Jahren keine Behandlungsstudien vor.

Schlüsselwörter Krafft-Ebing · Begutachtung $\cdot$ Zwang $\cdot$ BDSM $\cdot$ Rückfall $\cdot$ Behandlung

\section{Sexual sadism: present state of knowledge and coding according to DSM-5-TR and ICD-11}

\begin{abstract}
The term sexual sadism describes the lustful arousal through experiencing control or causing humiliation or pain to another person. Nowadays, psychiatric classification manuals focus on coercion and non-consent in order to distinguish the forensic variant of sexual sadism from its consensual counterpart of sado-masochistic role play (bondage discipline/dominance submission/sadism masochism, BDSM). Furthermore, sexual sadism has to be differentiated from so-called trait sadism or everyday sadism as a personality trait. The present article elucidates the modern nosology of forensically relevant, coercive sexual sadism disorder according to ICD-11. The sexual sadism scale is described as a diagnostic aid for expert witness assessment. Results on criminal recidivism are highlighted. The diagnosis as such is not significantly associated with violent or sexual reoffending. Nonetheless, there is a strong link with most grievous index offenses (i.e., sexually motivated homicide). As current literature reviews show, there are no treatment studies available from recent years.
\end{abstract}

Keywords Krafft-Ebing · Assessment $\cdot$ Coercion $\cdot$ BDSM $\cdot$ Reoffending $\cdot$ Treatment

Univ.-Prof. Dr. phil. habil. Andreas Mokros

andreas.mokros@fernuni-hagen.de

1 LG Persönlichkeits-, Rechtspsychologie und Diagnostik,

FernUniversität in Hagen, 58084 Hagen, Deutschland

2 Bezirkskrankenhaus Straubing, Straubing, Deutschland 


\section{Einleitung}

\section{Begriffsgeschichte}

In der Genealogie der Moral schrieb Nietzsche (1954 [1887]):

Leiden-sehn tut wohl, Leiden-machen noch wohler - das ist ein harter Satz, aber ein alter mächtiger menschlich-allzumenschlicher Hauptsatz, den übrigens vielleicht auch schon die Affen unterschreiben würden: denn man erzählt, daß sie im Ausdenken von bizarren Grausamkeiten den Menschen bereits reichlich ankündigen und gleichsam „vorspielen“. Ohne Grausamkeit kein Fest: so lehrt es die älteste, längste Geschichte des Menschen - und auch an der Strafe ist so viel Festliches! (S. 807)

Was Nietzsche als allgemein menschliche Neigung beschreibt, nämlich die Bereitschaft, sich am Schmerz eines anderen erfreuen zu können (und deren harmlos-alltägliche Ausprägungsformen möglicherweise Schadenfreude und abwärts gerichtete soziale Vergleiche sind), soll offenbar auch der Antrieb dafür sein, sich Grausamkeiten und Strafen auszudenken, die in ihrer Ritualisierung gleichsam etwas Festliches hätten. Dieses Motiv verortet Nietzsche so tief in der menschlichen Natur, dass es angeblich auch bei anderen Primaten vorhanden sei.

Tatsächlich ist die Geschichtsschreibung voller Beispiele für beide Phänomene: die individuelle Bereitschaft, Genuss aus dem Leiden eines anderen zu ziehen, und die Neigung, sich bizarre Grausamkeiten auszudenken. Der Psychiater Weitbrecht (1973) schrieb:

Die wollüstige Erregung beim Anhören von scheußlichen Mordtaten oder beim Beschauen von Bildern, die bis zum Orgasmus gesteigerte sexuelle Erregung vor allem von Frauen beim Zuschauen von qualvollen Hinrichtungen sind aus zahlreichen Berichten und Beobachtungen bekannt. Ein Blut- und Sexualrausch kann ganze Gruppen mit Windeseile überfallen und zu Orgien des genußvollen Abschlachtens von Opfern im Ausbruch ,spontanen Volkszornes“ bei Revolutionen und Pogromen führen. (S. 156, Hervorhebung im Original)

Den Autoren des vorliegenden Beitrags ist es nicht gelungen, historisch verbürgte Beispiele von „,bis zum Orgasmus gesteigerte[r] sexuelle[r] Erregung vor allem von Frauen beim Zuschauen von qualvollen Hinrichtungen“, wie Weitbrecht (1973) schrieb, zu finden, trotz der angeblich ,,zahlreichen [Berichte] und Beobachtungen". Allerdings kann wohl die Schilderung der Ermordung von Prinzessin Marie Louise von Savoyen im Zuge der Septembermassaker von 1792 als Beleg für die Verbindung von Grausamkeit, sexueller Erniedrigung und Schaulust dienen: Nachdem die Prinzessin getötet worden war, schnitt ihr angeblich jemand den Mons pubis ab und hielt ihn sich zum Spektakel der Menge wie einen Schnurrbart vor das Gesicht (McCallam 2007).

Interessanterweise erschien nur ein Jahr vor Nietzsches oben genannter Schrift eine Monografie, die in Teilen in lateinischer Sprache verfasst war, um eine „wollüstige Erregung" bei der Lektüre jedenfalls unter Nichtfachleuten zu verhindern: Richard von Krafft-Ebings Psychopathia sexualis (1886). Darin nahm von Krafft-Ebing u. a. auch das Phänomen des sog. Lustmords in den Blick; in späteren Auflagen des Werks war im selben Kapitel dann von $\mathrm{Sa}$ dismus die Rede. Von Krafft-Ebing (1886, 1907) zufolge sei es erwiesen, dass Begierde und Grausamkeit häufig zusammen auftreten würden. Gleichzeitig warnte von KrafftEbing davor, bei Folter, Hinrichtung oder dergleichen vorschnell eine sadistische Motivation zu unterstellen:

Es wäre jedoch durchaus falsch und übertrieben, überall da, wo ausserordentliche, überraschende Grausamkeit sich findet, diese aus sadistischer Perversion erklären zu wollen, und, wie es hie und da geschieht, in den zahllosen Greueln der Geschichte oder auch in gewissen massenpsychologischen Erscheinungen der Gegenwart den Sadismus als Motiv vorauszusetzen. [...]

Daneben ist noch ein starkes psychisches Element zu berücksichtigen, welches namentlich die Anziehungskraft erklärt, die heute noch Hinrichtungen u. dgl. ausüben; das ist die Lust am starken und ungewöhnlichen Eindruck überhaupt, am seltenen Schauspiel, der gegenüber das Mitleid in rohen oder abgestumpften Naturen schweigt. (S. 96-97)

Sadismus beschrieb von Krafft-Ebing $(1886,1907)$ u. a. wie folgt: „Sadismus ist also nichts anderes als eine pathologische Steigerung von - andeutungsweise auch unter normalen Umständen möglichen - Begleiterscheinungen der psychischen Vita sexualis, insbesondere der Männlichen, ins Masslose und Monströse“ (S. 69). Die Begriffe „Sadist“ oder ,sadistisch“" wurden allerdings nicht durch von KrafftEbing geprägt, sondern tauchten bereits zuvor im Schrifttum des 19. Jahrhunderts auf (Reinhardt 2014). Der Wortstamm bezieht sich dabei auf Donatien Alphonse François, Marquis de Sade (1740-1814), der in seinen Schriften (u. a. Justine) eben solche Akte sexueller Demütigung und Quälerei beschrieben hat, was seinen Biografen zufolge offenbar auch seiner gelebten Sexualität entsprach (Reinhardt 2014).

Oftmals wird Sadismus stereotyp mit der Zufügung von Schmerzen (etwa durch Schlagen) in Verbindung gebracht, auch wenn dies nur eine von vielen Möglichkeiten ist, um Dominanz und Kontrolle zu erleben (Karpman 1954). Dabei hat bereits von Krafft-Ebing $(1886,1907)$ auf die Viel- 
zahl möglicher Verhaltensweisen hingewiesen, die durchaus auch ideelle oder symbolische Formen beinhalten könnten: „So entsteht eine lange Reihe von Formen, welche mit den schwersten Verbrechen beginnt und bei läppischen Handlungen endigt, die dem perversen Bedürfnisse des Sadisten eine bloss symbolische Befriedigung gewähren sollen“ (S. 70). Wie knapp 100 Jahre später MacCulloch et al. (1983) dargelegt haben, sei vielmehr der übersteigerte Wunsch nach dem Erleben von Kontrolle das maßgebliche Motiv:

... it is precisely the wish to control that is the primary motivating force in sadism, and because there is a range of degrees and kinds of control which can be applied by one person to another, sadism may manifest itself in a variety of ways. [...] The range of controlling behaviour under consideration forms a continuum from subtle verbal control through various types of psychological control to actual physical intervention such as bondage, imprisonment, hypnosis, anaesthesia and even blows to render the victim unconscious or dead. (S. 20-21, Hervorhebung im Original)

\section{Abgrenzung des Konstrukts}

\section{Einvernehmliche sadomasochistische Rollenspiele}

Neben der forensisch-psychiatrisch relevanten Erscheinungsform des Sexualsadismus, die hier im Vordergrund steht, gibt es auch eine mutmaßlich in der Allgemeinbevölkerung relativ weit verbreitete Neigung, sexuell-sadistische Praktiken einvernehmlich auszuleben, also mit einem einwilligenden (und zur Einwilligung befähigten) Gegenüber. Gemäß gängigen Erscheinungsformen wie Fesseln und Disziplinierung (,,bondage“ und ,discipline“), Dominanz und Unterwerfung (,dominance“ und ,submission“) und Sadismus und Masochismus im Sinne von Schmerz, Leiden und Demütigung (,sadism“ und „,masochism“) bezeichnet man diese Spielart sexueller Betätigung oder Neigung mit dem Kürzel BDSM.

Allgemein orientiert sich die BDSM-Subkultur an der Maßgabe: ,safe, sane and consensual“ - also sicher, vernünftig und einvernehmlich (Dunkley und Brotto 2020). Das schließt aber natürlich nicht aus, dass es zu gelegentlichen Übertretungen vorher vereinbarter Grenzen kommen kann. Auch ist es plausibel, dass die BDSM-Subkultur eine besondere Anziehungskraft auf Menschen mit bestimmten Persönlichkeitsakzentuierungen hat, etwa im Sinne des Narzissmus (was den dominanten Part anbelangt) oder im Sinne von Borderline- oder dependenten Strukturen (was den submissiven Part betrifft). Im Übrigen ist die Rollenfestlegung als Dom (im Englischen: „top“) oder Sub bzw. Sklave/Sklavin (im Englischen auch: ,bottom“) nicht in al- len Fällen festgelegt; manche wechseln mitunter die Rollen und werden als ,switch“ (oder „switcher") bezeichnet. Schließlich ist festzuhalten, dass es einen Graubereich gibt, in dem die vermeintlich selbst gewählte submissive Rolle die Folge massiver Einwirkung eines Straftäters sein kann (Hazelwood et al. 1993).

Im Allgemeinen ist aber davon auszugehen, dass BDSM, abgesehen von Einzelfällen, kein nennenswertes Thema für die forensische Psychiatrie (i. S. von Begutachtung oder Behandlung) ist. Demensprechend haben Vertreterinnen und Vertreter der BDSM-Subkultur erreicht, dass beispielsweise in Schweden und Finnland die Diagnose Sadomasochismus nicht mehr Bestandteil der nationalen Versionen der International Klassifikation psychischer Störungen, 10. Auflage (World Health Organization 2004), gewesen ist (Helsingin Sanomat 2011). Solche Bestrebungen mögen im Sinne der Destigmatisierung sexueller Präferenzvarianten von psychisch gesunden Menschen sein; in forensisch-psychiatrischer Hinsicht stellen sie eine unzulässige Übernahme dar, weil der Begriff (im Sinne von Krafft-Ebings) ja ein originär forensisches Konzept widerspiegelt. Eine aktuelle Übersichtsarbeit zum Thema BDSM stammt von De Neef et al. (2019). Eine Verhaltenscheckliste für entsprechende sexuelle Praktiken und Vorlieben haben Weierstall und Giebel (2017) vorgelegt.

\section{Charakter- bzw. Alltagssadismus}

Im Diagnostischen und Statistischen Manual Psychischer Störungen der Amerikanischen Psychiatrischen Vereinigung, 3. revidierte Auflage (DSM-IIIR; Wittchen et al. 1989) war die sog. sadistische Persönlichkeitsstörung als probatorische Kategorie im Anhang gelistet, wurde aber in nachfolgenden Auflagen des DSM nicht mehr berücksichtigt (Stone 1998), auch weil keine hinreichende Differenzierung gegenüber der antisozialen Persönlichkeitsstörung gegeben war. Maßgeblicher Unterschied zum sexuellen Sadismus war, dass sexuelle Empfindung für die Diagnose der sadistischen Persönlichkeitsstörung keine Rolle gespielt hat. $\mathrm{Zu}$ den diagnostischen Kriterien für die sadistische Persönlichkeitsstörung gehörten u.a.: andere Menschen in Gegenwart anderer zu erniedrigen oder zu beschämen; sich am seelischen oder körperlichen Leid einer anderen Person zu erfreuen; eine Faszination für Gewalt, Waffen, Kampfsportarten, Verletzungen oder Folter (Deutsche Gesellschaft für Sexualforschung 2007).

In einer Stichprobe von 166 Gutachtensfällen von Sexualmördern aus dem Hamburger Institut für Sexualforschung und Forensische Psychiatrie wurde die Konkordanz der sadistischen Persönlichkeitsstörung mit der Diagnose des sexuellen Sadismus untersucht (Hill et al. 2006). Unter den Sexualmördern mit der Diagnose Sexualsadismus lag die Konkordanzrate mit der sadistischen Persönlichkeitsstö- 
rung bei 36,1\% (22 von 61 Fällen); dieser Wert lag deutlich oberhalb der Auftretensrate der sadistischen Persönlichkeitsstörung unter den übrigen Sexualmördern ohne die Diagnose sexueller Sadismus (6,7 \% bzw. 7 von 105 Fällen). Unter 70 Sexualstraftätern aus einer sozialtherapeutischen Anstalt in Wien lag die Konkordanzrate beider Diagnosen bei 18,6\% (13 Fälle; Berner et al. 2003).

Im Rahmen subklinischer Persönlichkeitsausprägungen wird eine Entsprechung der sadistischen Persönlichkeitsstörung mittlerweile als Alltags- oder Charaktersadismus bezeichnet und unter die sog. Dunkle Tetrade der Persönlichkeit subsumiert (Paulhus 2014; Paulhus und Dutton 2016). Eine Erfassungsskala, die auch in deutscher Sprache vorliegt, ist das Comprehensive Assessment of Sadistic Tendencies (CAST; Voggeser und Göritz 2016). Ein Verhaltenskorrelat von hohen Ausprägungen an Alltagssadismus ist das Trolling im Internet (Buckels et al. 2014).

\section{Nosologie}

\section{Diagnostisches und Statistisches Manual psychischer Störungen}

Im DSM-5 (American Psychiatric Association 2015) wird allgemein zwischen Paraphilien und paraphilen Störungen unterschieden. Ist die sexuelle Präferenz für mindestens ein halbes Jahr auf das Leiden (psychisch oder physisch) einer anderen Person ausgerichtet, was sich in entsprechenden Vorstellungen, intensiven Wünschen oder einschlägigen Verhaltensweisen manifestiert, dann ist das sog. A-Kriterium erfüllt (Sadismus); hat derjenige deswegen gegen andere gehandelt, die nicht darin eingewilligt haben, oder leidet selbst beruflich, sozial oder anderweitig unter seinen Fantasien oder Bedürfnissen, dann ist auch das sog. B-Kriterium erfüllt (sexuell sadistische Störung). Zudem ist aufzuschlüsseln, ob sich die betroffene Person in einer kontrollierten Umgebung befindet, wodurch die Gelegenheit, sexuell-sadistische Verhaltensweisen zu zeigen, eingeschränkt sein kann. Und schließlich besteht die Möglichkeit zu codieren, dass sich die Störung in völliger Remission befindet, nämlich dann, wenn seit mindestens 5 Jahren auBerhalb eines reglementierten Settings weder entsprechende Handlungen noch einschlägige Bedürfnisse aufgetreten sind, die zu Leiden oder Beeinträchtigungen aufseiten des Betroffenen geführt hätten.

Einschränkend muss erwähnt werden, dass wesentliche Aspekte beider diagnostischer Kriterien des DSM-5 von außen nicht beobachtbar sind, sondern allenfalls indirekt aus dem Verhalten des Betroffenen abgeleitet werden können, sofern derjenige nicht gewillt ist, seine Fantasien oder Bedürfnisse mitzuteilen. Eine vielversprechende Ergänzung zur psychiatrischen Exploration, die allerdings noch weite- rer Überprüfung bedarf, stellt daher ein indirektes Testverfahren auf Grundlage der Betrachtungszeit für entsprechendes Bildmaterial dar (Larue et al. 2014). Ferner sind Blickbewegungsmessungen im Hinblick auf einschlägige Stimuli erprobt worden (Hogue und Perkins 2014). Solange solche technischen Messverfahren noch nicht hinreichend auf Objektivität, Reliabilität und Validität geprüft sowie normiert sind, bleibt die kriteriengeleitete Verhaltensbeurteilung die Methode der Wahl (s. Abschn. „Diagnostik“).

In der bevorstehenden Textrevision des DSM-5, dem DSM-5-TR, stehen keine Veränderungen an den diagnostischen Kriterien an. Lediglich die Erläuterungen zur Prävalenz, zu Entstehung und Verlauf, zu Risiko- bzw. prognostischen Faktoren sowie zur Differenzialdiagnostik und zu Komorbiditäten wurden auf der Grundlage von Literaturrecherchen aktualisiert. Das Ergebnis dieser systematischen Literaturrecherchen wurde von Mokros et al. (2019) veröffentlicht.

\section{Internationale Klassifikation psychischer Störungen}

Die 11. Auflage der International Classification of Diseases (ICD-11) wurde im Juni 2018 durch die Weltgesundheitsorganisation präsentiert und soll am 01.01.2022 in Kraft treten (Bundesinstitut für Arzneimittel und Medizinprodukte o.J.). Die bislang nur in englischer Sprache vorhandene ICD-11-Definition für die Coercive sexual sadism disorder (im Deutschen am ehesten: zwangsweise sexuell-sadistische Störung) lautet wie folgt (World Health Organization 2018):

Coercive sexual sadism disorder is characterised by a sustained, focused and intense pattern of sexual arousal—as manifested by persistent sexual thoughts, fantasies, urges or behaviours-that involves the infliction of physical or psychological suffering on a non-consenting person. In addition, in order for Coercive Sexual Sadism Disorder to be diagnosed, the individual must have acted on these thoughts, fantasies or urges or be markedly distressed by them. Coercive Sexual Sadism Disorder specifically excludes consensual sexual sadism and masochism.

Bemerkenswert an der Neufassung der Diagnose ist dreierlei:

- die Abkehr von der vorherigen Verschmelzung von Sadismus und Masochismus (Sadomasochismus) zu einer einzigen Störungskategorie, wie dies in der ICD-10 der Fall war (World Health Organization 2004);

- der Fokus auf nichteinvernehmliche Erscheinungsformen (und somit die Abgrenzung von BDSM), was auch durch 
die Hinzufügung des Adjektivs „coercive“ (auf Deutsch: zwangsweise) nochmals betont wird;

- die Nichtberücksichtigung einer ungestörten Variante, wie dies im DSM-5 mit der Paraphilie (gegenüber der paraphilen Störung) der Fall ist.

\section{Diagnostik}

\section{Sexuelle Sadismus-Skala}

Verschiedenen Untersuchungen zufolge (z.B. Marshall et al. 2002) erwies sich die Beurteilerübereinstimmung im Hinblick auf die Diagnose sexueller Sadismus, und somit deren Reliabilität, als unzureichend (für eine Übersicht: Nitschke et al. 2013). Auch aus diesem Grund stellten Marshall und Hucker (2006) auf Grundlage einer Expertenbefragung eine aus 17 diagnostisch relevanten (Tat-)Verhaltensweisen bestehende Liste zusammen. Durch empirische Untersuchungen konnte daraus schließlich eine aus 11 Kriterien bestehende Skala entwickelt werden (10 Kriterien aus der Liste von Marshall et al. sowie ein Item gemäß Knight et al. 1998), die Sexuelle SadismusSkala (SeSaS; Mokros et al. 2014):

\section{Sexuelle Sadismus-Skala}

- Item 1: sexuelle Erregung bei den Tathandlungen,

- Item 2: Machtausübung, Kontrolle, Dominanz,

- Item 3: Quälen des Opfers,

- Item 4: erniedrigendes, demütigendes Verhalten gegenüber dem Opfer,

- Item 5: Verstümmelung von Geschlechtsorganen,

- Item 6: Verstümmelung anderer Körperstellen oder -teile,

- Item 7: Ausüben von exzessiver physischer Gewalt,

- Item 8: Einführen von Gegenständen in Körperöffnungen des Opfers,

- Item 9: ritualisierte Handlungen,

- Item 10: Einsperren des Opfers/räumliche Nötigung,

- Item 11: Mitnahme von Trophäen (aus: Nitschke et al. 2020).

In einer Auswertung von 4 Studien wies die SeSaS bei einem Schwellenwert von 4 erfüllten Kriterien eine diagnostische Sensitivität von $95 \%$ sowie eine Spezifität von $99 \%$ auf (Nitschke et al. 2013). Details zur Entwicklung sowie zu den psychometrischen Gütekriterien der SeSaS sind beispielsweise dem Buchbeitrag von Yoon et al. (2019) zu entnehmen. Ein Manual (SeSaS), das einerseits die Kriterien der Skala definiert und andererseits weitere klinische Aspekte der Diagnostik von sexuellem Sadismus berücksichtigt, befindet sich in der Entwicklung (Nitschke et al. 2020). Ein Vorteil der SeSaS ist der Fokus auf beobachtba- rem bzw. dokumentiertem Verhalten. Die einzelnen Kriterien beziehen sich auf Tathergangsvariablen und nicht auf subjektive Angaben von Personen, die situativ bedingt (beispielsweise bei Begutachtungen) Symptome dissimulieren könnten. Zur Informationsgewinnung können somit schriftliche Urteilsbegründungen, Vorgutachten oder Zeugenaussagen dienen. Hierbei ist zu beachten, dass die $\mathrm{SeSaS}$ ein zusätzliches Hilfsmittel zur Diagnostik darstellt, jedoch die eigentliche klinische Diagnose keinesfalls ersetzen soll und darf.

\section{Begutachtung}

\section{Schuldfähigkeit}

Bei der Beurteilung der Schuldfähigkeit im Erkenntnisverfahren besteht im Hinblick auf schwerste Sexualdelikte u. U. die Annahme, dass die Diagnose eines sexuellen Sadismus geradezu regelhaft mit dem Vorliegen der psychiatrischen Voraussetzungen für eine erhebliche Verminderung der Steuerungsfähigkeit einhergehe. Aus diesem Grund ist darauf hinzuweisen, dass auch für die Diagnose sexueller Sadismus diejenigen Kriterien gelten, die bei der Begutachtung anderer Paraphilien gültig sind. Nach den Empfehlungen einer interdisziplinären Expertenkommission (Boetticher et al. 2007) muss z.B. unbedingt geprüft werden, wie groß der Anteil des Sexualsadismus an der gesamten Sexualstruktur des Probanden ist. Weitere Kriterien sind u. a. die Prüfung der Integration der Störung der Sexualpräferenz in das Persönlichkeitsgefüge oder das Ausmaß, in dem sexuell-sadistische Impulse in der Vergangenheit kontrolliert werden konnten. Somit ist die Schwelle, ab der sexueller Sadismus dem Eingangsmerkmal einer schweren anderen seelischen Abartigkeit zugewiesen werden kann, keineswegs willkürlich und geht weit über die Bewertung der Schwere einer Tat hinaus. Für eine ausführliche Darstellung der Beurteilung der Schuldfähigkeit im Hinblick auf Störungen der Sexualpräferenz sei auf die Novemberausgabe 2020 dieser Zeitschrift mit dem Schwerpunktthema Verminderte Schuldfähigkeit verwiesen, speziell auf den Artikel zur operationalisierten, kriteriengeleiteten Beurteilung der Schuldfähigkeit bei paraphilen Störungen (Dobbrunz und Briken 2020).

\section{Kriminalprognose}

In einer Metaanalyse werteten Eher et al. (2016, Studie 1) insgesamt 7 Studien mit einem Gesamtstichprobenumfang von 2169 Personen aus. Danach war das relative Risiko (RR) für erneute Gewalt- einschließlich Sexualstraftaten unter Probanden mit der Diagnose Sexualsadismus zwar erhöht $(\mathrm{RR}=1,18)$, aber nicht in einem signifikanten Maße 
(zweiseitiges $p=0,170$ ). Auch im Hinblick auf Sexualrückfälle $(\mathrm{RR}=1,38)$ war die Erhöhung nicht statistisch bedeutsam. Betrachtete man für das erste Kriterium (erneute Gewalt- einschließlich Sexualdelikte) jedoch nur jene 5 Studien, in denen gemäß dem DSM-IV codiert worden war, erwies sich das RR $(1,29)$ durchaus als signifikant erhöht (zweiseitiges $p=0,044$ ). Die Befundlage ist also nicht ganz eindeutig.

In einer weiteren Analyse werteten Eher et al. (2016, Studie 2) für eine der bereits zuvor berücksichtigten Stichproben $(n=768)$ aus, ob im Rahmen einer sog. Cox-Regression bei variablem Tatgelegenheitszeitraum die DSM-IVDiagnose mit Rückfälligkeit zusammenhängt, wenn man Kovariaten wie Alter bei Entlassung, Psychopathie (PCLR) oder aktuarisches Risiko (SORAG, Static-99) kontrolliert. Das Alter bei Entlassung erwies sich in Verbindung mit jedem anderen Prädiktor (PCL-R, SORAG und Static99) als statistisch bedeutsam, nicht aber mit der Ausprägung von sexuellem Sadismus - weder als kategoriale Diagnose (DSM-IV) noch als kontinuierlicher Kennwert (Se$\mathrm{SaS}$ ). Demnach trug Sexualsadismus nichts zur Varianzaufklärung hinsichtlich Rückfälligkeit bei, wenn gängige Risikofaktoren bereits berücksichtigt waren. Dieser Befund ist zwischenzeitlich durch eine weitere Studie aus der Schweiz repliziert worden (Gonçalves et al. 2020).

Bevor aber hinsichtlich der Diagnose des sexuellen Sadismus vorschnell Entwarnung gegeben wird, gilt es, auf folgende Punkte hinzuweisen: PCL-R, SORAG und Static-99 stellen valide Instrumente für die Kriminalprognose dar. Die zusätzliche Diagnose eines sexuellen Sadismus führte in bisherigen Studien nicht dazu, eine bereits durch solche etablierten Kriminalprognoseinstrumente ermittelte ungünstige Legalprognose noch ungünstiger erscheinen zu lassen. Zudem sind bei der Legalprognoseforschung in Bezug auf sexuellen Sadismus mögliche methodische Verzerrungen zu berücksichtigen. Täter mit der betreffenden Diagnose dürften seltener, später oder mitunter auch gar nicht entlassen werden (Berner u. Briken 2010), und zwar umso mehr, je schwerwiegender das Indexdelikt war. Indexdelikte von Sexualsadisten sind jedoch tendenziell besonders schwerwiegend, was sich aus dem starken Zusammenhang der Diagnose mit sexuell motivierten Tötungsdelikten ergibt (Stefańska et al. 2019).

\section{Behandlungsprognose}

Wie die systematische Literaturrecherche von Mokros et al. (2019) gezeigt hat, gibt es keine aktuellen Behandlungsstudien zur forensisch-relevanten zwangsweisen sexuell-sadistischen Störung. Die meisten der wenigen Behandlungsstudien stammen aus den 1960er- und 1970er-Jahren und beziehen sich auf rein verhaltenstherapeutische Interventionen wie aversive Konditionierung (Laws et al. 1978; Marks et al. 1970; Mees 1966) oder verdeckte Desensibilisierung (Davison 1968; Hayes et al. 1978). Die Aussagekraft dieser Veröffentlichungen ist zudem dadurch eingeschränkt, dass es sich bei 3 Artikeln lediglich um Berichte über Fallstudien handelt (Davison 1968; Laws et al. 1978; Mees 1966). In einer neueren Arbeit aus den 2000er-Jahren wurden u. a. auch Patienten mit der Diagnose sexueller Sadismus mit selektiven Serotonin-Wiederaufnahmehemmern (SSRI) behandelt (Kraus et al. 2007); allerdings beinhaltete das Studiendesign keine Kontrollgruppe, sodass mögliche Effekte nicht auf die Medikation zurückgeführt werden können. Daher kann - wie vor gut 10 Jahren bereits von Berner und Briken (2010) in dieser Zeitschrift in ähnlicher Weise empfohlen - weiterhin nur auf klinische Erfahrung und Plausibilität rekurriert werden. Eine Praxisleitlinie im Sinne eines Behandlungsalgorithmus, abhängig von der Intensität einer sexuellen Präferenzstörung, haben Berner et al. (2007) vorgelegt. Sexueller Sadismus wird diesem Algorithmus zufolge als gefährlichere Paraphilie eingeordnet und eine medikamentöse Behandlung mit Cyproteronacetat (CPA) empfohlen. Sollte die Medikation unzureichend wirken oder es zu Leberfunktionsstörungen kommen, solle auf ein Medikament aus der Klasse der Luteinisierendes-Hormon-Releasing-Hormon(LHRH)-Agonisten umgestellt werden. Bei unzureichender Wirkung von CPA oder des LHRH-Agonisten, insbesondere bei gleichzeitiger depressiver, ängstlicher oder zwanghafter Symptomatik, wird die zusätzliche Gabe eines SSRI empfohlen. Sollte sich ein Risiko für einen Anabolikamissbrauch zeigen, könne der LHRH-Agonist auch mit CPA kombiniert werden. Ausdrücklich empfahlen Berner et al. (2007), sich nicht auf eine pharmakologische Therapie zu beschränken, sondern gleichzeitig mit dem Patienten auch psychotherapeutisch zu arbeiten.

\section{Fazit}

Der Begriff Sadismus taucht in unterschiedlichen Bedeutungen auf. Maßgeblich für den forensisch-psychiatrischen Bereich ist die zwangsweise sexuell-sadistische Störung (gemäß ICD-11) bzw. die sexuell-sadistische Störung (gemäß DSM-5/DSM-5-TR), in Abgrenzung zum einvernehmlichen Sadomasochismus (BDSM) sowie zum sog. Charakter- oder Alltagssadismus. Aufgrund der Hinweise auf eine geringe Reliabilität der klinischen Diagnose empfiehlt sich die Orientierung an Tatverhaltenskriterien (konkret: $\mathrm{SeSaS}$ ) als Hilfsmittel für die Klassifikation. Ist das Rückfallrisiko eines Probanden bereits durch einschlägige aktuarische Prognoseverfahren abgebildet, ist das zusätzliche Risiko, das mit sexuellem Sadismus einhergeht, offenbar gering; dies bedeutet aber nicht, dass sexueller Sadismus für die Gefährlichkeitsbeurteilung irrelevant wäre, denn der Zusammenhang mit besonders schwerwiegenden Indexdelikten (kon- 
kret: sexuell-motivierte Tötungsdelikte) ist überproportional stark. Im Hinblick auf die Schuldfähigkeitsbegutachtung ist auf die Rolle des sexuellen Sadismus im Persönlichkeitsgefüge und der Sexualstruktur des Probanden zu achten und nicht ausschließlich auf die Tatschwere zu fokussieren. Behandlungsempfehlungen ergeben sich, ohne systematische Evidenz, im Sinne einer Praxisleitlinie zur Kombination triebdämpfender Medikation mit psychotherapeutischer Intervention.

Funding Open Access funding enabled and organized by Projekt DEAL.

Interessenkonflikt A. Mokros war Berater für die Erstellung der Textrevision des DSM-5 (DSM-5-TR) der American Psychiatric Association im Hinblick auf die sexuell-sadistische Störung. A. Mokros und J. Nitschke sind (Ko-)Autoren der Sexuellen Sadismus-Skala (SeSaS) sowie der deutschen Version der Hare Psychopathy Checklist-Revised (PCL-R).

Open Access Dieser Artikel wird unter der Creative Commons Namensnennung 4.0 International Lizenz veröffentlicht, welche die Nutzung, Vervielfältigung, Bearbeitung, Verbreitung und Wiedergabe in jeglichem Medium und Format erlaubt, sofern Sie den/die ursprünglichen Autor(en) und die Quelle ordnungsgemäß nennen, einen Link zur Creative Commons Lizenz beifügen und angeben, ob Änderungen vorgenommen wurden.

Die in diesem Artikel enthaltenen Bilder und sonstiges Drittmaterial unterliegen ebenfalls der genannten Creative Commons Lizenz, sofern sich aus der Abbildungslegende nichts anderes ergibt. Sofern das betreffende Material nicht unter der genannten Creative Commons Lizenz steht und die betreffende Handlung nicht nach gesetzlichen Vorschriften erlaubt ist, ist für die oben aufgeführten Weiterverwendungen des Materials die Einwilligung des jeweiligen Rechteinhabers einzuholen.

Weitere Details zur Lizenz entnehmen Sie bitte der Lizenzinformation auf http://creativecommons.org/licenses/by/4.0/deed.de.

\section{Literatur}

American Psychiatric Association (2015) Diagnostisches und Statistisches Manual Psychischer Störungen - DSM-5. Hogrefe, Göttingen (deutsche Ausgabe herausgegeben von Peter Falkai und Hans-Ulrich Wittchen, mitherausgegeben von Manfred Döpfner, Wolfgang Gaebel, Wolfgang Maier, Winfried Rief, Henning Saß und Michael Zaudig)

Berner W, Briken P (2010) Sexueller Sadismus und Sexualkriminalität. Forens Psychiatr Psychol Kriminol 4:90-97. https://doi.org/ 10.1007/s11757-010-0048-5

Berner W, Berger P, Hill A (2003) Sexual sadism. Int J Offender Ther Comp Criminol 47:383-395. https://doi.org/10.1177/ $0306624 X 03256131$

Berner W, Hill A, Briken P, Kraus C, Lietz K (2007) Behandlungsleitlinie: Störung der sexuellen Präferenz. In: Gaebel W, Falkai P (Hrsg) Praxisleitlinien in Psychiatrie und Psychotherapie, Bd. 8. Steinkopff, Darmstadt

Boetticher A, Nedopil N, Bosinski HAG, Saß H (2007) Mindestanforderungen an Schuldfähigkeitsgutachten. Forens Psychiatr Psychol Kriminol 1:3-9. https://doi.org/10.1007/s11757-006-0002-8

Buckels EE, Trapnell PD, Paulhus DL (2014) Trolls just want to have fun. Pers Individ Differ 67:97-102. https://doi.org/10.1016/j.paid. 2014.01.016
Bundesinstitut für Arzneimittel und Medizinprodukte (o.J.) FAQ: Wann kommt die ICD-11? https://www.dimdi.de/dynamic/de/ faq/faq/Wann-kommt-die-ICD-11/. Zugegriffen: 4. Dez. 2020

Davison GC (1968) Elimination of a sadistic fantasy by a client-controlled counterconditioning technique: a case study. J Abnorm Psychol 73:84-90. https://doi.org/10.1037/h0025440

Deutsche Gesellschaft für Sexualforschung - DGfS (2007) Störungen der sexuellen Präferenz. Steinkopff, Darmstadt

Dobbrunz S, Briken P (2020) Operationalisierte, kriteriengeleitete Beurteilung der Schuldfähigkeit bei paraphilen Störungen - Stand und Ausblick. Forens Psychiatr Psychol Kriminol 14:430-443. https://doi.org/10.1007/s11757-020-00633-w

Dunkley CR, Brotto LA (2020) The role of consent in the context of BDSM. Sex Abuse 32:657-678. https://doi.org/10.1177/ 1079063219842847

Eher R, Schilling F, Hansmann B, Pumberger T, Nitschke J, Habermeyer E, Mokros A (2016) Sexual sadism and violent reoffending. Sex Abuse 28:46-72. https://doi.org/10.1177/1079063214566715

Gonçalves LC, Rossegger A, Gerth J, Singh JP, Endrass J (2020) Sexual sadism among sex offenders in Switzerland. Sex Abuse 32:79-100. https://doi.org/10.1177/1079063218800473

Hayes SC, Brownell KD, Barlow DH (1978) The use of self-administered covert sensitization in the treatment of exhibitionism and sadism. Behav Ther 9:283-289. https://doi.org/10.1016/S00057894(78)80114-2

Hazelwood R, Warren J, Dietz P (1993) Compliant victims of the sexual sadist. Austr Fam Phys 22:474-479

Helsingin Sanomat-International Edition (2011) Transvestism is no longer a disease in Finland. http://www.hs.fi/english/article/ Transvestism+is+no+longer+a+disease+in+Finland/1135266192 528 (Erstellt: 16. Mai 2011). Zugegriffen: 13. Juli 2012

Hill A, Habermann N, Berner W, Briken P (2006) Sexual sadism and sadistic personality disorder in sexual homicide. J Personal Disord 20:671-684. https://doi.org/10.1521/pedi.2006.20.6.671

Hogue T, Perkins D (2014) Violence is in the eye of the beholder: using eye-tracking to understand sexual aggression. 13th International Conference der International Association for the Treatment of Sexual Offenders (IATSO), Porto, Portugal, 09.2014 (Vortrag)

Karpman B (1954) The sexual offender and his offenses: etiology, pathology, psychodynamics, and treatment. I: review of the literature (1912-1951). II: psychodynamics of sexual offenses and a formulation of the problem. Julian Press, New York

Knight RA, Warren J, Reboussin R, Soley BJ (1998) Predicting rapist type from crime-scene variables. Crim Justice Behav 25:46-80. https://doi.org/10.1177/0093854898025001004

von Krafft-Ebing R (1886) Psychopathia sexualis. Enke, Stuttgart

von Krafft-Ebing R (1907) Psychopathia sexualis mit besonderer Berücksichtigung der konträren Sexualempfindung: Eine medizinisch-gerichtliche Studie für Ärzte und Juristen, 13. Aufl. Enke, Stuttgart

Kraus C, Strohm K, Hill A, Habermann N, Berner W, Briken P (2007) Selektive Serotoninwiederaufnahmehemmer (SSRI) in der Behandlung von Paraphilien. Fortschr Neurol Psychiatr 75:351-356. https://doi.org/10.1055/s-2006-944261

Larue D, Schmidt AF, Imhoff R, Eggers K, Schönbrodt FD, Banse R (2014) Validation of direct and indirect measures of preference for sexualized violence. Psychol Assess 26:1173-1183. https:// doi.org/10.1037/pas0000016

Laws DR, Meyer J, Holmen ML (1978) Reduction of sadistic sexual arousal by olfactory aversion: a case study. Behav Res Ther 16:281-285. https://doi.org/10.1016/0005-7967(78)90026-8

MacCulloch MJ, Snowden PR, Wood PJ, Mills HE (1983) Sadistic fantasy, sadistic behaviour and offending. Br J Psychiatry 143:20-29. https://doi.org/10.1192/bjp.143.1.20

Marks I, Gelder M, Bancroft J (1970) Sexual deviants two years after electric aversion. Br J Psychiatry 117:173-185. https://doi.org/10. 1192/bjp.117.537.173 
Marshall WL, Hucker SJ (2006) Severe sexual sadism: Its features and treatment. In: McAnulty RD, Burnette MM (Hrsg) Sexual deviation and sexual offenses. Sex and sexuality, Bd. 3. Praeger, Westport, S 227-250

Marshall WL, Kennedy P, Yates P, Serran GA (2002) Diagnosing sexual sadism in sexual offenders: reliability across diagnosticians. Int J Offender Ther Comp Criminol 46:668-677. https://doi.org/ $10.1177 / 0306624 x 02238161$

McCallam D (2007) „La machine“: Sade, the guillotine, and eroticism. In: Burwick F, Tucker K (Hrsg) Marquis de Sade and the scientia and techne of eroticism. Cambridge Scholars Publishing, Newcastle, S 54-71

Mees HL (1966) Sadistic fantasies modified by aversive conditioning and substitution: a case study. Behav Res Ther 4:317-320. https:// doi.org/10.1016/0005-7967(66)90028-3

Mokros A, Schilling F, Weiss K, Nitschke J, Eher R (2014) Sadism in sexual offenders: evidence for dimensionality. Psychol Assess 26:138-147. https://doi.org/10.1037/a0034861

Mokros A, Wessels J, Hofmann M, Nitschke J (2019) Coercive sexual sadism: a systematic qualitative review. Curr Psychiatry Rep 21:135. https://doi.org/10.1007/s11920-019-1118-9

De Neef N, Coppens V, Huys W, Morrens M (2019) Bondage-discipline, dominance-submission and sadomasochism (BDSM) from an integrative biopsychosocial perspective: a systematic review. Sex Med 7:129-144. https://doi.org/10.1016/j.esxm.2019.02.002

Nietzsche F (1954) Zur Genealogie der Moral, Zweite Abhandlung „Schuld“, „Schlechtes Gewissen“ und Verwandtes. Werke in drei Bänden, Bd. 2. Hanser, München (Original erschienen 1887)

Nitschke J, Mokros A, Osterheider M, Marshall WL (2013) Sexual sadism: Current diagnostic vagueness and the benefit of behavioral definitions. Int J Off Ther Comp Criminol 57:1441-1453. https:// doi.org/10.1177/0306624X12465923

Nitschke J, Schilling F, Eher R, Mokros A (2020) SESAS - Sexual Sadism Scale: Manual zur Erfassung von Merkmalen, die auf das Vorliegen eines sexuellen Sadismus hinweisen [Unveröffentlichtes Manuskript]. Vorab erhältlich vom Erstautor via Email: j.nitschke@bkh-straubing.de

Paulhus DL (2014) Toward a taxonomy of dark personalities. Curr Dir Psychol Sci 23:421-426. https://doi.org/10.1177/0963721414547 737
Paulhus DL, Dutton DG (2016) Everyday sadism. In: Zeigler-Hill V, Marcus DK (Hrsg) The dark side of personality: science and practice in social, personality, and clinical psychology. American Psychological Association, Washington, S 109-120

Reinhardt V (2014) De Sade oder die Vermessung des Bösen: Eine Biographie. C. H. Beck, München

Stefańska EB, Nitschke J, Carter AJ, Mokros A (2019) Sadism among sexual homicide offenders: validation of the Sexual Sadism Scale. Psychol Assess 31:132-137

Stone MH (1998) Sadistic personality in murderers. In: Millon T, Simonsen E, Birket-Smith M, Davis RD (Hrsg) Psychopathy: antisocial, criminal and violent behavior. Guilford, New York, S 346-355

Voggeser B, Göritz AS (2016) CAST in German: validating a German version of the comprehensive assessment of sadistic tendencies. 50th Annual Conference of the German Psychological Society (DGPs), Leipzig, Germany (Poster)

Weierstall R, Giebel G (2017) The Sadomasochism Checklist: a tool for the assessment of sadomasochistic behavior. Arch Sex Behav 46:735-745. https://doi.org/10.1007/s10508-016-0789-0

Weitbrecht HJ (1973) Psychiatrie im Grundriss, 3. Aufl. Springer, Berlin, Heidelberg, New York

Wittchen HU, Sass H, Zaudig M, Koehler K (1989) Diagnostisches und statistisches Manual psychischer Störungen DSM-III-R. Beltz, Weinheim

World Health Organization (2018) International classification of diseases for mortality and morbidity statistics (11th Revision). https://icd.who.int/browse11/1-m/en. Zugegriffen: 4. Dez. 2020

World Health Organization (2004) International statistical classification of diseases and health related problems, tenth revision, Vol. F, 2. Aufl. WHO Press, Genf

Yoon D, Mauzaite A, Mokros A (2019) Sexual Sadism Scale. In: Milhausen RR, Sakaluk JK, Fisher TD, Davis CM, Yarber WL (Hrsg) Handbook of sexuality-related measures, 4. Aufl. Routledge, New York, S 525-527 